\title{
Leukapheresis Does Not Improve Early Survival Outcome of Acute Myeloid Leukemia with Leukostasis Patients - A Dual-Center Retrospective Cohort Study
}

\author{
Ikhwan Rinaldi ${ }^{1}$ \\ Resti Mulya Sari ${ }^{2}$ \\ Vanya Utami Tedhy ${ }^{3}$ \\ Kevin Winston (D) $^{3}$ \\ 'Division of Hematology and Medical \\ Oncology, Department of Internal \\ Medicine, Cipto Mangunkusumo National \\ General Hospital, Faculty of Medicine, \\ Universitas Indonesia, Jakarta, Indonesia; \\ ${ }^{2}$ Division of Hematology and Medical \\ Oncology, Department of Internal \\ Medicine, Dharmais National Cancer \\ Hospital, Faculty of Medicine, Universitas \\ Indonesia, Jakarta, Indonesia; ${ }^{3}$ Faculty of \\ Medicine, Universitas Indonesia, Jakarta, \\ Indonesia
}

Introduction: Leukostasis is a medical emergency with high mortality which often occurs in acute myeloid leukemia patients with hyperleukocytosis. One of the therapies that can be used for leukostasis in acute myeloid leukemia is leukapheresis. However, whether leukapheresis can provide better survival benefit when compared with patients not receiving leukapheresis is still unclear. Hence, we aimed to evaluate the effect of chemotherapy plus leukapheresis combination versus chemotherapy only on 28-day survival of acute myeloid leukemia patients with leukostasis. Methods: This study was a dual-center retrospective cohort using secondary data from medical records collected from November 2018 to March 2019. Inclusion criteria were adult patients aged 18 years old or above, diagnosed with acute leukemia with hyperleukocytosis status defined by WBC count greater than 100,000/uL, and with symptoms of leukostasis. One-month survival analysis was conducted using Kaplan-Meier curve method. Univariate and multivariate analyses were then conducted using Cox proportional hazards model to obtain value of hazard ratio (HR) with a $95 \%$ confidence interval (CI).

Results: A total of 38 patients were obtained for analysis. The median overall survival was 25 days (95\% CI: 17.001-32.999 days) in the chemotherapy only group and 20 days (95\% CI: 1.497-38.503) in the chemotherapy with leukapheresis group. The use of leukapheresis did not affect 28-day survival (HR: 1.140; 95\% CI: 0.396-3.283; p value: 0.809 ) and 7-day survival (HR: 1.073 ; 95\% CI: $0.277-4.152$; p value: 0.919 ). In the multivariate analysis, age $\geq 60$ years, blast percentage $\geq 90 \%$, creatinine $\geq 1.4 \mathrm{mg} / \mathrm{dL}$, and presence of disseminated intravascular coagulation were associated with worse 28 -day survival.

Conclusion: AML patients with leukostasis who received both chemotherapy and leukapheresis did not have better 28-day survival and 7-day survival when compared with patients receiving chemotherapy only. Old age, high blast percentage, high creatinine, and presence of disseminated intravascular coagulation were prognostic factors for worse 28-day survival. Keywords: leukemia, hyperleukocytosis, leukostasis, leukapheresis, chemotherapy, survival

\section{Introduction}

Leukostasis is a medical emergency which can occur in acute leukemia due to obstruction of small blood vessels by malignant blast cells which result in tissue and organ ischemia with high potential for mortality and morbidity. ${ }^{1-3}$ Diagnosis of leukostasis is clinically based on manifestations arising from tissue hypoxia in the target organs such as respiratory distress, impaired kidney function, central nervous system disorders, and coagulopathy, after excluding other possible etiologies. ${ }^{2,3}$
Division of Hematology and Medical

Oncology, Department of Internal

Medicine, Cipto Mangunkusumo National

General Hospital, Faculty of Medicine,

Universitas Indonesia, Jakarta, 10430,

Indonesia

Tel +628I I I00I758

Email ikhwan.rinaldi@ui.ac.id 
Leukostasis generally starts to occur in conditions such as hyperleukocytosis where the leucocyte count is above 100,000 cells $/ \mu \mathrm{L}$ in acute myeloid leukemia (AML) and in range of around $200,000 / \mu \mathrm{L}$ to $400,000 / \mu \mathrm{L}$ in acute lymphoblastic leukemia (ALL). ${ }^{4-6}$ The difference in the cutoff numbers of leukocytes between AML and ALL is due to the fact that blast cells from myeloid progenitor have bigger size and lower deformability than blast cells from lymphoid progenitor. ${ }^{3}$ However, leukostasis may also occur in leucocyte count below 100,000 cells/ $\mu \mathrm{L} .^{3}$

The mortality from leukostasis in acute leukemia reaches about $40 \%$, which usually occurs within a few weeks of diagnosis. ${ }^{3}$ Hence, prompt treatment of acute leukemia patients with leukostasis is important to reduce mortality and prevent complications. The aim of leukostasis therapy is to reduce the number of blast cells, which can be achieved by using either chemotherapy with or without leukapheresis. ${ }^{2,7}$ Chemotherapy works by causing blast cells to undergo apoptosis in infiltrated organs, peripheral circulation, and bone marrow, thereby reducing the total burden of leukocytes and resolving leukostasis. ${ }^{3}$ The type of chemotherapy given is specific according to the type of leukemia, hence the administration is usually postponed until the type of leukemia is confirmed. One of the chemotherapies that can be used is hydroxyurea. ${ }^{3}$ However, the issue with chemotherapy in acute leukemia patients with leukostasis is the potential risk of tumor lysis syndrome (TLS) after chemotherapy due to large number of blast cells undergoing lysis simultaneously., $3,8,9$ Additionally, not all patients are clinically suitable to receive chemotherapy, such as due to old age or frailty.

Another treatment modality for leukostasis is leukapheresis, in which the blast cells from the circulation are removed through a filter of the leukapheresis machine. ${ }^{10}$ Leukapheresis can reduce the number of blast cells faster but the procedure is associated with the risk of blast cells' rebound because the removal of blast cells is not accompanied by destruction of blast cells in the bone marrow which can quickly replace the removed blast cells in peripheral circulation. ${ }^{11,12}$ Other disadvantages of leukapheresis procedure are the expensive cost, the requirement of the availability of special facilities, insertion of central venous catheter, and the need for experienced staff. ${ }^{2,13}$ The main advantage of leukapheresis is being able to overcome metabolic disorders and coagulation via plasma and electrolyte administration during the procedure. ${ }^{2,13}$

Whether adding leukapheresis treatment in conjunction with chemotherapy can produce mortality reduction in acute leukemia patients with leukostasis is currently still unclear and contradictory in several studies. ${ }^{3,10,12-18}$ For example, there were several studies supporting the use of leukapheresis such as a study conducted in 2017 by Nan et al, which observed that the use of leukapheresis decreased 28-day-mortality rate in AML patients with hyperleukocytosis when compared with patients not receiving leukapheresis $(30.8 \%$ vs $57.7 \%$, p: 0.022). ${ }^{19}$ Another study by Bug et al also stated that leukapheresis improved survival in the first 3 weeks of AML patients (16\% vs 32\%, p: 0.015). ${ }^{16}$ Meanwhile other studies found no benefit of early mortality from the use of leukapheresis such as the study by Malkan et al, Porcu et al, Giles et al, and Stahl et al. ${ }^{13,15,20,21}$

The difference between the results of various studies indicates that there is still unclear evidence that supports the use of leukapheresis in reducing mortality of acute leukemia patients with leukostasis. Additionally, many studies were different in the measured survival endpoint. Therefore, this research was conducted with the aim to analyze and compare 7-day survival, 14-day survival, 21-day survival, and 28-day survival outcomes of acute myeloid leukemia patients with leukostasis who received leukapheresis compared to those who did not receive it.

\section{Methods Study Design}

This was a dual-center retrospective cohort study using secondary data from medical records to compare 7-day, 14-day, 21-day, and 28-day survival outcome of acute myeloid leukemia patients with leukostasis based on the type of treatments received. The treatments were divided into leukapheresis plus chemotherapy group and chemotherapy only group.

The research was conducted at Cipto Mangunkusumo National General Hospital and Dharmais National Cancer Hospital using medical record data of inpatient acute myeloid leukemia patients with leukostasis during 2007-2018. Both hospitals are tertiary hospital and national referral hospital in Indonesia. Medical record data were searched and gathered by the authors starting from November 2018 to March 2019.

\section{Patients}

The target population was Indonesian acute myeloid leukemia patients who underwent treatment at Cipto Mangunkusumo National General Hospital and Dharmais National Cancer Hospital during 2007-2018. Inclusion 
criteria used were adult patients aged 18 years old or above suffering from acute myeloid leukemia with hyperleukocytosis of WBC count greater than 100,000/uL, with symptoms of leukostasis. Exclusion criteria for the study were incomplete medical records, acute leukemia type other than AML, AML without hyperleukocytosis, AML without leukostasis, palliative leukemia patients, and refractory leukemia patients.

The type of leukemia was diagnosed from examination of bone marrow biopsy. Hyperleukocytosis status was diagnosed from blood leukocyte count. Leukostasis status was diagnosed clinically by the attending physicians in our centers based on the clinical manifestations associated with organ ischemia in patients with hyperleukocytosis. Patients suspected of having leukostasis were also given a series of diagnostic tests such as chest X-ray, blood cultures, brain imaging, cerebrospinal fluid analysis, liver enzymes, lactic dehydrogenase, and coagulation tests to exclude other etiologies before making leukostasis diagnosis. In this study, the chemotherapy used for AML patients consisted of either hydroxyurea or cytarabine.

Demographic and clinical data of patients were taken from medical records for baseline characteristics. Baseline characteristics presented in this study included age, gender, BMI, clinical manifestations type of leukostasis, presence of chronic comorbidities, and hematological parameters. Hematological parameters taken included hemoglobin, leukocytes, platelet count, creatinine, and blast percentage. Chronic comorbidities included tuberculosis infection, chronic liver disease, diabetes mellitus, history of stroke, chronic heart failure, hypertension, and chronic kidney disease. Tumor lysis syndrome events and disseminated intravascular coagulation events prior to treatments were also recorded.

\section{Procedure of Leukapheresis}

Patients were first informed about leukapheresis procedure and possible adverse effects that may arise from the procedure. The patients were then given time to ask questions or reconsider his or her decision of undergoing leukapheresis. Written informed consent was then obtained prior to procedure. In our centers, central venous catheter placement was conducted on all patients. No peripheral venous catheter was used for the procedure. The leukapheresis procedures for all patients were conducted using continuous-flow blood cell separator (Haemonetics ${ }^{\circledR}, \mathrm{MCS}+{ }^{\circledR}, \mathrm{LN}$ 9000 apheresis machine). The collection speed was 20 $300 \mathrm{cc} /$ minute. An average of $15 \%$ to $20 \%$ of a patient's total blood volume was processed during a single leukapheresis session with a total average leukapheresis duration of five hours or until reaching target of buffy coat. During the procedure, acid citrate dextrose solution A (ACD-A) was used as an anticoagulant with concomitant administration of intravenous calcium gluconate.

\section{Endpoints}

Primary endpoint of this study was 7-day, 14-day, 21-day and 28-day survival after starting treatments. Possible confounding factors were analyzed by multivariate analysis to determine their association with survival.

\section{Ethics}

The research protocol was approved by the Faculty Health Research Ethics Committee Medicine, University of Indonesia Cipto Mangunkusumo National General Hospital (FKUI-RSCM) No. 1209/UN2.F1/ETIK/2018. All patients' medical record data entered into research were protected to maintain confidentiality. Since this was a retrospective cohort study, no informed consent was needed. Finally, this study is in compliance with Declaration of Helsinki.

\section{Statistical Analysis}

All the statistical analyses were performed using SPSS (SPSS 21, IBM) and STATA (MP 14.2, Stata Corp LP, College Station, TX, USA). Data on the baseline characteristics of the subjects at the time of the intervention were described in the tables. Numerical data with a normal distribution were displayed as means and standard deviation while numerical data with skewed distribution were displayed as median with minimum-maximum range value. Normal distribution of data was assessed with Kolmogorov-Smirnov test. Meanwhile, categorical data were described as percentage. Differences in baseline characteristics were assessed using Chi-squared test or Fisher's exact test for categorical variables and $t$-test or Mann-Whitney $U$ test for continuous variables.

Survival analysis and comparison of 7-day, 14-day, 21day, and 28-day survival of AML patients with both hyperleukocytosis and leukostasis based on type of treatments received were analyzed using Kaplan-Meier survival curve method. Differences in survival curves were then measured with log rank (Mantel-Cox) and Breslow (Generalized Wilcoxon) tests. P values of $<0.05$ were considered to be statistically significant.

For the main variables which were associated with one-month survival in this study, univariate analysis was 
conducted using Cox proportional hazards model to obtain value of hazard ratio (HR) with a 95\% confidence interval (CI). Variables selected for univariate cox regression analysis were filtered with proportional hazard assumption tests. The type of proportional hazard assumption tests used for the study consisted of Ln Ln survival test and global test. Variables fulfilled proportional hazard assumption test if there was no intersect on Ln Ln survival test and had $p$ value of $>0.05$ on global test. Subsequently, variables analyzed in univariate analysis that had $p$ value of $\leq 0.25$ were selected for multivariate analysis.

\section{Results}

\section{Baseline Characteristics}

A total of 80 adult acute leukemia patients were initially identified from the medical records. However, 18 patients were excluded due to lack of leukostasis symptoms. Additionally, 6 patients were excluded due to lack of bone marrow biopsy data and another 18 patients were excluded due to being ALL type. As a result, the number of subjects used in this study was 38 AML patients with leukostasis (Table 1).

From the 38 AML patients with leukostasis, a total of 11 patients $(28.9 \%)$ received chemotherapy with leukapheresis while the other 27 patients (71.1\%) received chemotherapy only. The baseline characteristics of AML patients can be seen in Table 1 .

Mortality rates within the first month were compared weekly. Results showed that there was no association between 7-day mortality and the type of treatment given (p: 1.000). A total of 7 patients died in the first week and eleven in the fourth week for the chemotherapy group. Meanwhile, in the chemotherapy plus leukapheresis group, a total of three patients died in the first week and five in the fourth week. Moreover, no statistically significant differences in mortality were observed in the second to fourth week as well.

Table I Baseline Characteristics of AML Patients

\begin{tabular}{|c|c|c|c|c|}
\hline Characteristics & Total $n=38$ & $\begin{array}{c}\text { Leukapheresis + } \\
\text { Chemotherapy } n=1 \text { I }\end{array}$ & $\begin{array}{l}\text { Chemotherapy Only } \\
\qquad n=27\end{array}$ & $P$ value \\
\hline Age (years) (SD) & $42.45(15.52)$ & $40.82(8.67)$ & 43.11 (I7.67) & 0.685 \\
\hline Gender & & & & 0.762 \\
\hline Male (\%) & $17(44.7 \%)$ & $4(36.4 \%)$ & $13(48.1 \%)$ & \\
\hline Female (\%) & $21(55.3 \%)$ & 7 (63.6\%) & $14(51.9 \%)$ & \\
\hline Body Mass Index $\left(\mathrm{Kg} / \mathrm{m}^{2}\right)$ (median) & $21.125(11.98-36.57)$ & $21.96(11.98-30.08)$ & 21.71 (12.33-36.57) & 0.552 \\
\hline Hemoglobin $(g / d l)(S D)$ & $7.527(2.227)$ & $6.694(2.165)$ & $7.866(2.200)$ & 0.144 \\
\hline MCV (Femtolitre) (SD) & $87.458(5.689)$ & $87(7.1255)$ & $87.644(5.140)$ & 0.756 \\
\hline Leukocyte $\left(\mathrm{mm}^{3}\right)$ (median) & $\begin{array}{c}205,835(105,000- \\
847,000)\end{array}$ & $\begin{array}{c}353,830(143,360- \\
847,000)\end{array}$ & $\begin{array}{c}170,930(105,000- \\
369,100)\end{array}$ & 0.003 \\
\hline Thrombocyte $\left(\mathrm{mm}^{3}\right)$ (median) & $39,550(6000-665,000)$ & $57,900(6000-125,000)$ & $34,000(7540-665,000)$ & 0.509 \\
\hline Blast (\%) (median) & $90(42-97)$ & $90(54-97)$ & $90(42-96)$ & 0.485 \\
\hline Creatinine (mg/dl) (median) & $1.08(0.46-6.00)$ & $1.4 \mid(0.56-5.48)$ & $1.03(0.46-6.00)$ & 0.573 \\
\hline Respiratory leukostasis (\%) & $21(55.3 \%)$ & $2(18.2 \%)$ & 19 (70.4\%) & 0.010 \\
\hline Central Nervous System Leukostasis (\%) & $20(52.6 \%)$ & $5(45.5 \%)$ & $15(55.6 \%)$ & 0.836 \\
\hline Cardiovascular Leukostasis (\%) & $10(26.3 \%)$ & $3(27.3 \%)$ & 7 (25.9\%) & 1.000 \\
\hline Gastrointestinal Leukostasis (\%) & $22(57.9 \%)$ & $4(36.4 \%)$ & $18(66.7 \%)$ & 0.176 \\
\hline Tumor Lysis Syndrome (\%) & 15 (39.5\%) & $3(27.3 \%)$ & $12(44.4 \%)$ & 0.538 \\
\hline Disseminated Intravascular Coagulation (\%) & $13(34.2 \%)$ & $3(27.3 \%)$ & $10(37 \%)$ & 0.843 \\
\hline Chronic Comorbidities (\%) & $14(36.8 \%)$ & $2(18.2 \%)$ & $12(44.4 \%)$ & 0.250 \\
\hline
\end{tabular}




\section{Survival Outcomes}

The median overall survival was 25 days (95\% CI: $17.001-$ 32.999 days) in the chemotherapy only group and 20 days (95\% CI: 1.497-38.503) in the chemotherapy with leukapheresis group. At day 28, a total of 11 of the 27 patients receiving chemotherapy only (41.7\%) and 5 of the 11 patients receiving chemotherapy with leukapheresis $(45.5 \%)$ had died.

The 28-day survival was not statistically better in patients receiving chemotherapy with leukapheresis group than in chemotherapy only group (p Log Rank: 0.806; p Breslow: 0.827) (Figure 1). The hazard ratio for chemotherapy plus leukapheresis group when compared with chemotherapy only group for 28-day survival was 1.140 (95\% CI: 0.396-3.283; p value: 0.809 ). Similarly, based on Kaplan-Meier curves, the survival rates of patients receiving chemotherapy plus leukapheresis were also not statistically

A

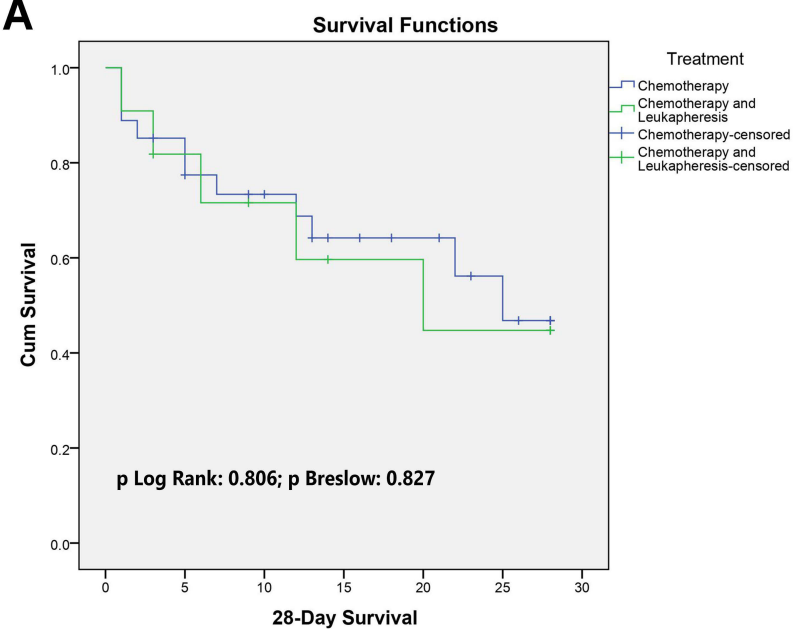

C

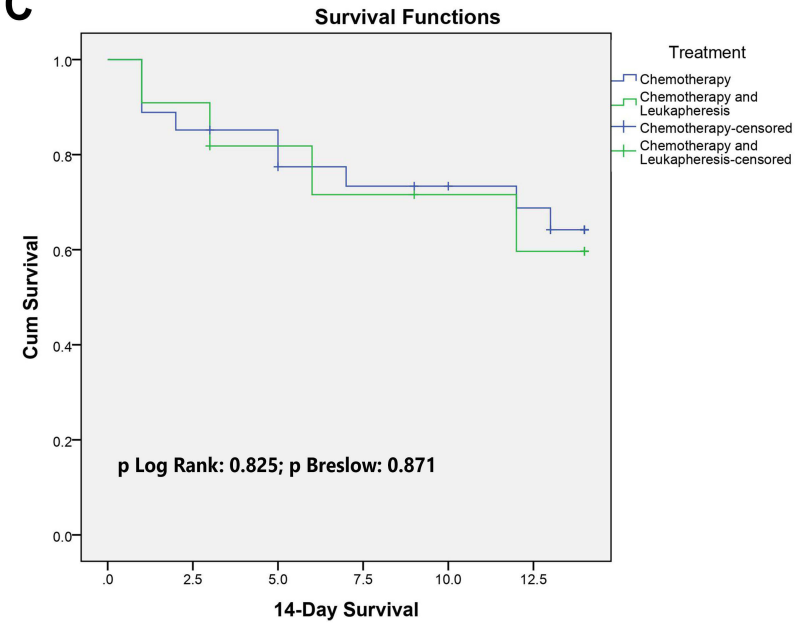

better at day-7 (p Log Rank: 0.528; p Breslow: 0.733), day14 (p Log Rank: 0.825; p Breslow: 0.871), and day-21 (p Log Rank: 0.917; p Breslow: 0.949) when compared with their respective chemotherapy only groups (Figure 1). The hazard ratio for chemotherapy plus leukapheresis group when compared with chemotherapy only group for 7-day survival was 1.073 (95\% CI: 0.277-4.152; p value: 0.919 ).

\section{Univariate and Multivariate Analysis}

Univariate and multivariate analyses were conducted for 28day survival and 7-day survival. In 28-day survival, the use of chemotherapy plus leukapheresis did not statistically improve 28-day survival (HR: 1.140; 95\% CI: 0.3963.283; p: 0.809). Other factors such as age, blast percentage, creatinine level, presence of gastrointestinal leukostasis, TLS, DIC, and chronic comorbidities in univariate analysis
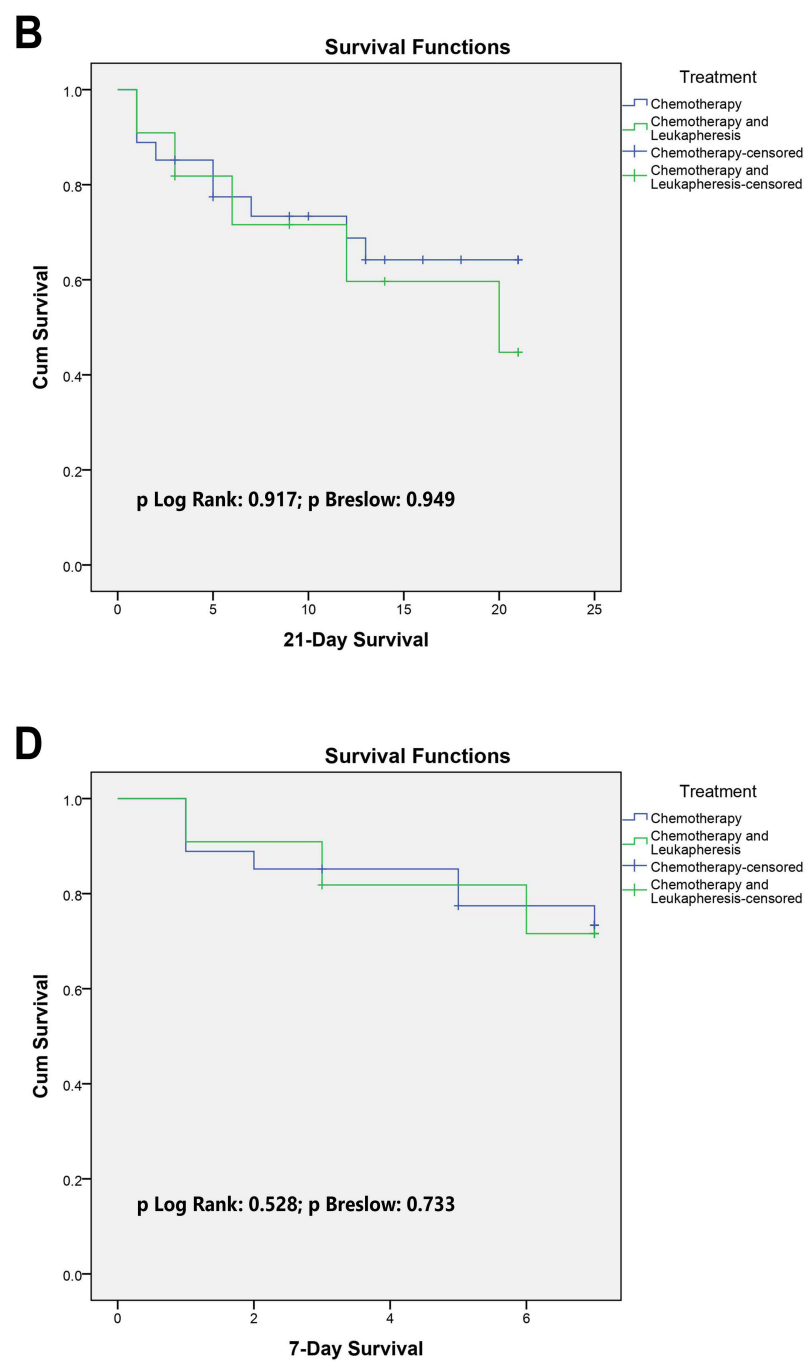

Figure I Survival of acute myeloid leukemia patients with leukostasis based on chemotherapy plus leukapheresis treatment and chemotherapy only treatment. (A) 28 Days; (B) 21 days; (C) 14 days; (D) 7 days. 
also did not affect 28-day survival significantly (Table 2). However, patients with BMI of $\geq 25$ were shown to have worse survival (HR: 3.292; 95\% CI: 1.180-9.183; p: 0.023) in the univariate analysis. Variables from the univariate analysis which fulfilled proportional hazard assumption test and thus were selected for multivariate analysis can be seen in Supplementary Table S1. In the multivariate analysis, age $\geq 60$ years, blast percentage $\geq 90 \%$, creatinine $\geq 1.4$, and presence of DIC were associated with worse 28-day survival (Table 2). Hence, BMI no longer remained statistically significant in the multivariate analysis.

\section{Discussion}

\section{Acute Myeloid Leukemia and Leukostasis}

In AML, there is an unregulated clonal proliferation of immature blast cells with concomitant deterioration of bone marrow function. AML is currently the most common leukemia in adults. Left untreated, up to $20 \%$ of de novo AML patients progress to hyperleukocytosis state defined as leukocyte count above $>100000 / \mu \mathrm{L}^{3,12,22}$ Although hyperleukocytosis is a laboratory abnormality, hyperleukocytosis is a very important clinical entity when encountered by physicians due to numerous high-mortality-complication $\mathrm{s}$ that can arise such as leukostasis, TLS, and DIC.

Leukostasis is a clinical manifestation of organ ischemia due to intravascular lumen obstruction by immature blast cells which often occurs in hyperleukocytosis and is associated with significant mortality. ${ }^{2,23,24}$ The diagnosis of leukostasis is generally made clinically and empirically based on the clinical manifestations associated with organ ischemia in patients with hyperleukocytosis, after excluding other potential etiologies. ${ }^{25}$ For example, if an AML patient with hyperleukocytosis suddenly develops dyspnea, the clinician must first be able to exclude other potential causes of dyspnea before making a diagnosis of leukostasis. ${ }^{12,25}$ Hence, patients suspected of having leukostasis are often given a series of diagnostic tests such as chest X-ray, blood cultures, brain imaging, cerebrospinal fluid analysis, liver enzymes, lactic dehydrogenase, and peripheral blood morphology.

Organs most commonly affected by leukostasis are the CNS and lungs. The exact reasons why these organs have a tendency to be affected by leukostasis is currently unknown, however, it can be speculated that the rich vasculature and the physiological functions of CNS and

Table 2 Univariate Analysis and Multivariate Analysis for 28-Day Survival of Acute Myeloid Leukemia Patients

\begin{tabular}{|c|c|c|c|c|c|c|c|}
\hline \multirow[t]{2}{*}{ Variable } & \multirow[t]{2}{*}{ Category } & \multicolumn{3}{|c|}{ Univariate Analysis } & \multicolumn{3}{|c|}{ Multivariate Analysis } \\
\hline & & $\begin{array}{c}\text { Hazard } \\
\text { Ratio }\end{array}$ & $95 \% \mathrm{Cl}$ & $P$ value & $\begin{array}{c}\text { Hazard } \\
\text { Ratio }\end{array}$ & $95 \% \mathrm{Cl}$ & $P$ value \\
\hline Therapy & $\begin{array}{l}\text { Chemotherapy + leukapheresis } \\
\text { Chemotherapy }\end{array}$ & $\begin{array}{l}1.140 \\
1\end{array}$ & $0.396-3.283$ & 0.809 & - & - & - \\
\hline Age & $\begin{array}{l}\geq 60 \text { years } \\
<60 \text { years }\end{array}$ & $\begin{array}{l}2.513 \\
1\end{array}$ & $0.805-7.851$ & 0.113 & $\begin{array}{l}5.541 \\
I\end{array}$ & $|.031-29.78|$ & 0.046 \\
\hline BMI & $\begin{array}{l}\geq 25 \mathrm{~kg} / \mathrm{m}^{2} \\
<25 \mathrm{~kg} / \mathrm{m}^{2}\end{array}$ & $\begin{array}{l}3.292 \\
1\end{array}$ & $1.180-9.183$ & 0.023 & $\begin{array}{l}3.465 \\
1\end{array}$ & $0.934-12.959$ & 0.063 \\
\hline Blast & $\begin{array}{l}\geq 90 \% \\
<90 \%\end{array}$ & $\begin{array}{l}2.322 \\
1\end{array}$ & $0.747-7.224$ & 0.146 & $\begin{array}{l}6.058 \\
1\end{array}$ & $1.464-25.075$ & 0.013 \\
\hline Creatinine & $\begin{array}{l}\geq 1.4 \mathrm{mg} / \mathrm{dL} \\
<1.4 \mathrm{md} / \mathrm{dL}\end{array}$ & $\begin{array}{l}2.516 \\
1\end{array}$ & $0.925-6.844$ & 0.071 & $\begin{array}{l}5.749 \\
1\end{array}$ & $1.792-18.442$ & 0.003 \\
\hline Gastrointestinal Leukostasis & $\begin{array}{l}\text { Yes } \\
\text { No }\end{array}$ & $\begin{array}{l}1.567 \\
1\end{array}$ & $0.562-4.370$ & 0.391 & - & - & - \\
\hline Tumor Lysis Syndrome & $\begin{array}{l}\text { Yes } \\
\text { No }\end{array}$ & $\begin{array}{l}0.656 \\
1\end{array}$ & $0.226-1.902$ & 0.437 & - & - & - \\
\hline $\begin{array}{l}\text { Disseminated Intravascular } \\
\text { Coagulation }\end{array}$ & $\begin{array}{l}\text { Yes } \\
\text { No }\end{array}$ & $\begin{array}{l}2.005 \\
1\end{array}$ & $0.738-5.444$ & 0.172 & $\begin{array}{l}6.541 \\
I\end{array}$ & $1.822-23.490$ & 0.004 \\
\hline Chronic Comorbidities & $\begin{array}{l}\text { Yes } \\
\text { No }\end{array}$ & $\begin{array}{l}0.439 \\
1\end{array}$ & $0.141-1.369$ & 0.156 & - & - & - \\
\hline
\end{tabular}


lungs themselves, predispose these organs to disruption from leukostasis. The rich vasculature and high surface area of the lung for example, may increase the chance of blast cells to bind toward endothelium through E-selectin and vascular cell adhesion molecule-1 (VCAM-1). ${ }^{26,27}$ More studies are urgently needed to unravel the mechanisms in leukostasis initiation with the hope to discover a clinically relevant molecular target which can be used to prevent or remove leukostasis other than cytoreduction.

\section{Leukapheresis for AML Patients with Hyperleukocytosis}

Leukapheresis or leukocytapheresis is defined as removal of white blood cells from the blood by the apheresis machine through centrifugation. Other constituents of the blood are maintained and then infused back to the patient. Leukapheresis is one of the modalities available for management of hyperleukocytosis and leukostasis, not only in AML but also in ALL, chronic lymphocytic leukemia (CLL), and chronic myeloid leukemia (CML). ${ }^{6}$

Leukapheresis is widely accepted to be very effective in rapid reduction of white blood cell count with up to $70 \%$ of leukocytes removed in just a single session. ${ }^{28,29}$ However, like all medical treatments and procedures, there are always limitations. The major limitation of leukapheresis is that the procedure does not remove blast cells from bone marrow. ${ }^{30}$ These "leftover" blast cells in bone marrow may cause a short term rebound of hyperleukocytosis due to mobilization of blast cells from bone marrow into circulation which necessitates the use of chemotherapy to prevent rebound. ${ }^{4,13,30}$ Additionally, leukapheresis also does not remove organs' infiltrating blast cells. These blast cells then remain in affected organs and may continuously produce inflammatory response. ${ }^{30}$ Finally, already aggregated blast cells in intravascular lumen are also not removed. ${ }^{30}$ Hence, there appears to be some limitations of leukapheresis.

Another important factor that should be taken into consideration is that insertion of a central venous catheter is required for leukapheresis, which may cause several complications associated with catheter placement such as infection, pneumothorax, and bleeding. ${ }^{3,4}$ Furthermore, due to citrate being commonly used as anticoagulant during the procedure, hypocalcemia and its symptoms may occur during or after the procedure due to citrate's affinity to bind to calcium. $^{31,32}$ The most dangerous complication is QTc interval prolongation. ${ }^{32}$ Hence, periodic monitoring of the patient during and after the procedure is very important.

\section{Current Evidence on the Use of Leukapheresis for AML}

Currently, there are no randomized studies that evaluated the survival benefit of leukapheresis in AML patients with leukostasis. Furthermore, guidelines from professional medical organizations are scarce. For example, there are no guidelines from American Society of Hematology (ASH) and European Hematology Association (EHA) regarding whether to give leukapheresis or not. One of the guidelines currently available is 2013 Guidelines on the Use of Therapeutic Apheresis in Clinical Practice from American Society for Apheresis (ASFA) which stated that leukapheresis is indicated for symptomatic patients (Grade IB evidence) ${ }^{33}$ However, in the latest version of the guidelines in 2019, the grade of evidence was downgraded to IIB. ${ }^{34}$ Meanwhile, the grade of evidence for prophylactic leukapheresis is still IIC. ${ }^{33,34}$ Even now, all research on leukapheresis in AML come from observational studies.

In the literature, Ganzel et al stated that leukapheresis is recommended to be performed on all patients with hyperleukocytosis either with or without clinical manifestations to prevent imminent symptoms of leukostasis and to reduce the severity of tumor lysis syndrome., ${ }^{2,35}$ However, the effect of leukapheresis in improving mortality outcome is still controversial with studies producing conflicting results. ${ }^{13,15,16,19,36}$ For example, a retrospective study by Malkan et al with 28 AML subjects consisting of 10 subjects receiving leukapheresis and 18 subjects not receiving leukapheresis with outcome of early deaths in 15 days post-treatment, showed no difference in early death, although the study stated that leukapheresis can effectively lower plasma leukocytes. ${ }^{17}$ Other retrospective studies with bigger sample size such as by Pastore et al, with 52 AML patients, on the use of prophylactic leukapheresis and by Choi et al on therapeutic leukapheresis with 44 matched AML patients, also showed no difference in early mortality. ${ }^{10,36}$ Meanwhile, a study by Giles et al with a total of 146 AML patients showed that the use of leukapheresis reduced 2-week mortality. ${ }^{15}$ The study by Bug et al also supported that leukapheresis can reduce early 21-day mortality. ${ }^{16}$ It should be noted that although the study by Giles et al and Bug et al observed benefit in early mortality, no statistically significant difference was 
observed in long-term survival. ${ }^{15,16} \mathrm{~A}$ recent retrospective study by Göçer et al in 2021 found benefit in both early mortality and overall survival. ${ }^{37}$ However, differences in methodology and measured endpoint in these studies make it relatively difficult to interpret together.

A well-written systematic review and meta-analysis by Bewersdorf et al compared early mortality rates of AML patients treated with leukapheresis versus AML patients not receiving leukapheresis in 13 retrospective studies. ${ }^{38}$ The systematic review and meta-analysis found no shortterm benefit form leukapheresis and the authors discourage the use of routine leukapheresis for AML patients. ${ }^{38}$ However, due to all studies used being retrospective in design and including pediatric patients, there was mild heterogeneity in the pooled analysis. ${ }^{38}$ Given the rarity of AML patients with hyperleukocytosis, ethical issues, and preferences of clinicians in deciding which patients receive leukapheresis or not, it is theoretically very difficult for a randomized controlled study to be conducted. ${ }^{12}$

In Indonesia itself, leukapheresis was only introduced around the year 2002 and was only implemented as a means of therapy much later on. ${ }^{39}$ There were initially financial limitations, limited availability of blood components, as well as differences in knowledge of physicians and medical personnel regarding leukapheresis procedures in Indonesia that hindered the development of leukapheresis therapy. ${ }^{39}$ However, leukapheresis is now a common procedure in many Indonesian hospitals. The uncertainty of mortality benefit from leukapheresis is therefore a major concern not just for clinicians in Indonesia but also for clinicians worldwide in providing treatment for acute leukemia patients with leukostasis due to widespread availability of leukapheresis. Hence, this retrospective cohort study was conducted to measure early survival since leukostasis is an acute manifestation with significant early mortality and any important differences in survival should be detected within one month or less instead of a longer period.

\section{Baseline Characteristics}

Our research was conducted at Cipto Mangunkusumo National General Hospital and Dharmais National Cancer Hospital which recruited 38 acute myeloid leukemia patients with leukostasis for survival analysis. The mean age in all subjects was 42.45 years (Table 1). The mean age in treatment group and comparator group was similar (p: 0.685). When compared with other studies, the mean age in our study is younger. ${ }^{15-17,19}$ In terms of gender and
BMI, there were no significant differences between treatment and comparator group. However, the median baseline leukocyte count was considerably higher in chemotherapy plus leukapheresis group than chemotherapy only group $\left(353,830 / \mathrm{mm}^{3}\right.$ versus $170,930 / \mathrm{mm}^{3}$; $\mathrm{p}$ value: 0.003$)$. This difference also occurred in studies by Nan et al and Shallis et al. ${ }^{18,19}$ When compared with other studies, the overall median baseline leukocyte count of this study was higher. ${ }^{10,16,17}$ Finally, there was also significant difference in proportion of patients with pulmonary leukostasis between the two groups.

\section{Survival and Multivariate Analysis}

The one-month survival was not different between two groups (Figure 1). According to univariate Kaplan-Meier survival statistical analysis, there was no significant difference in 28-day survival (HR: 1.140; 95\% CI: $0.396-$ 3.283; $p$ value: 0.809 ) and 7-day survival (HR: $1.073 ; 95 \%$ CI: $0.277-4.152$; $p$ value: 0.919 ). Our results are in line with several studies. ${ }^{10,19-21,36,38}$ Despite similar conclusion, there were major differences in those studies that should be noted. For example, the study by Stahl et al used inclusion criteria of WBC $>50 \times 10^{9} / \mathrm{L}$ and also included patients without leukostasis, while all patients in this study had leukostasis symptoms. ${ }^{21}$ In contrast, the study by Ventura et al concluded that patients receiving leukapheresis had better mortality, however, it should be noted that this study was conducted more than several decades ago and in the study, non-leukostasis patients were more likely than leukostasis patients to receive leukapheresis. ${ }^{2}$ A retrospective study by Jin et al attempted to seek factors that influence efficacy of therapeutic leukapheresis in patients with hyperleukocytosis leukemia. ${ }^{29}$ In the study, the authors found that lymphocyte count, mean corpuscular hemoglobin $(\mathrm{MCH})$, and hematocrit levels prior to apheresis procedures were independent factors affecting survival. ${ }^{29}$ From the study by Jin et al, it can be speculated that there may have been a group of patients that received more benefit from leukapheresis based on lymphocyte count, $\mathrm{MCH}$, and hematocrit levels. Hence, further studies to elucidate and confirm whether there is a group of patients that would benefit from leukapheresis are needed.

In this study, leukapheresis and chemotherapy were given to patients with higher median leukocytes than patients receiving chemotherapy alone. From previous studies, it was found that leukocyte count is a prognostic factor for survival in AML. ${ }^{40-42}$ It is 
therefore possible that the higher median leukocyte count in leukapheresis and chemotherapy group affected the survival outcome in this study. The higher number of leukostasis manifestations in chemotherapy only group may also have affected the result by causing lower survival in the chemotherapy group, however, the leukostasis manifestations were not found to be prognostic factors in this study. Finally, many studies including this study recruited patients who were receiving leukapheresis therapy along with chemotherapy such as hydroxyurea or low-dose chemotherapy, resulting in difficulty to evaluate the effect of leukapheresis itself. ${ }^{4}$

The multivariate analysis in this study showed several interesting findings. The first was that age $\geq 60$ years old was associated independently with worse one-month survival (HR: 5.541; 95\% CI: 1.031-29.781; p: 0.046). This result is in concordance with the study by Liu et al, Kuo et al, and Kantarjian et al where age was a risk factor for early mortality. ${ }^{42-44}$ Older age is associated with worse physiological function and functional capacity, which may explain the finding of the multivariate analysis. ${ }^{7}$ Multiple comorbidities were also more commonly observed in the elderly. ${ }^{45}$ All of these factors may explain the lower survival in the elderly.

Our study also observed that creatinine of $\geq 1.4$ was associated with worse 28-day and 7-day survival. Similarly, the study by Pastore et al revealed that creatinine was associated with early death, but the confidence interval in the study was extremely wide. ${ }^{10}$ While the $95 \%$ $\mathrm{CI}$ in the multivariate analysis of this study was also wide, it was more precise than the study by Pastore et al. ${ }^{10}$ Other studies also supported the detrimental role of impaired kidney function in survival in AML patients. ${ }^{42,46}$ The exact reasons how kidney function impacts survival are currently unclear. We speculate that impaired kidney function is associated with poor performance status and other comorbidities which may indirectly decrease survival.

Another result from this study is that BMI $\geq 25$ was observed as prognostic factor for worse survival in the univariate analysis for 28-day survival and 7-day survival. However, BMI did not achieve statistical significance in multivariate analysis. The association between BMI and survival is in concordance with the study by Dhakal et al. ${ }^{47}$ However, the role of BMI in survival of acute leukemia patients is still unclear, as several studies show contradictory findings and the exact mechanisms of how BMI affects pharmacokinetics of treatments in acute leukemia are still under investigation. It is possible that BMI may affect the impact of pharmacokinetics of chemotherapy. ${ }^{48,49}$ Finally, both blast percentage $\geq 90 \%$ and presence of DIC were associated with worse 28-day survival.

\section{To Use or Not to Use Leukapheresis}

The question remains whether acute leukemia patients with hyperleukocytosis and leukostasis should receive leukapheresis or not. It is undeniable that leukapheresis is very effective in reducing the number of $\mathrm{WBC}$, however, leukapheresis does not remove aggregated blast cells in microcirculation or infiltrated tissues and hence may not improve survival, as there may still be tissue hypoxia and inflammation due to the leftover aggregated blast cells. ${ }^{4,30}$ Leukapheresis also confers procedural risk to patients especially those with comorbidities. ${ }^{12}$ Additionally, without removal of blast cells in bone marrow, patients with leukapheresis only treatment may have very high risk of relapse short-term. ${ }^{4}$ Hence, we do not recommend the addition of leukapheresis for AML patients with leukostasis, as its use together with chemotherapy does not provide better survival when compared with chemotherapy only, and there are many disadvantages of leukapheresis.

\section{Study Limitations}

There were several limitations in this study that should be taken into consideration when interpreting the results. First, we only had a limited sample size of 38 patients which may not be adequate for analyzing all confounders. Secondly, we also did not analyze the impact of cytogenetic and genetic mutation on survival. Finally, this was a retrospective cohort study which had all limitations associated with a cohort study.

\section{Conclusion}

AML patients with leukostasis who received both chemotherapy and leukapheresis did not have better 28-day survival and 7-day survival when compared with patients receiving chemotherapy only. Old age, high blast percentage, high creatinine, and presence of disseminated intravascular coagulation were prognostic factors for worse 28-day survival.

\section{Data Sharing Statement}

The datasets used and/or analyzed during the current study are available from the corresponding author on reasonable request. 


\section{Acknowledgments}

The authors wish to offer their appreciation to Jessica Novianto MD, Dewi Anggraeni MD, Lintang MD, Sabila MD, and Sarah MD for their tremendous help in this study.

\section{Author Contributions}

All authors made substantial contributions to conception and design, acquisition of data, or analysis and interpretation of data; took part in drafting the article or revising it critically for important intellectual content; agreed to submit to the current journal; gave final approval of the version to be published; and agree to be accountable for all aspects of the work.

\section{Funding}

All the study and collection, analysis, and interpretation of data and in writing the manuscript was funded by Cipto Mangunkusumo National General Hospital, Jakarta, Indonesia.

\section{Disclosure}

All authors declare that they have no conflict of interests for this work.

\section{References}

1. Giammarco S, Chiusolo P, Piccirillo N, et al. Hyperleukocytosis and leukostasis: management of a medical emergency. Expert Rev Hematol. 2017;10(2):147-154. doi:10.1080/17474086.2017.1270754

2. Ganzel C, Becker J, Mintz PD, Lazarus HM, Rowe JM. Hyperleukocytosis, leukostasis and leukapheresis: practice management. Blood Rev. 2012;26(3):117-122. doi:10.1016/j. blre.2012.01.003

3. Ali A, Mirrakhimov A, Abboud C, Cashen A. Leukostasis in adult acute hyperleukocytic leukemia: a clinician's digest. Hematol Oncol. 2016;34(2):69-78. doi:10.1002/hon.2292

4. Korkmaz S. The management of hyperleukocytosis in 2017: do we still need leukapheresis? Transfus Apher Sci. 2018;57(1):4-7. doi:10.1016/j.transci.2018.02.006

5. Barrett CL, Louw VJ, Webb MJ. Exchange transfusion as a life-saving intervention in three patients with different haematological malignancies with severe hyperleukocytosis where leukapheresis was not available. Transfus Apher Sci. 2013;49(3):397-402.

6. Aqui N, O'Doherty U. Leukocytapheresis for the treatment of hyperleukocytosis secondary to acute leukemia. Hematology Am Soc Hematol Educ Program. 2014;2014(1):457-460. doi:10.1182/asheducation-2014.1.457

7. Porcu P, Cripe LD, Ng EW, et al. Hyperleukocytic leukemias and leukostasis: a review of pathophysiology, clinical presentation and management. Leuk Lymphoma. 2000;39(1-2):1-18. doi:10.3109/ 10428190009053534

8. Howard SC, Jones DP, Pui C-H. The tumor lysis syndrome. $N$ Engl J Med. 2011;364(19):1844-1854. doi:10.1056/NEJMra0904569

9. Aslam HM, Zhi C, Wallach SL. Tumor lysis syndrome: a rare complication of chemotherapy for metastatic breast cancer. Cureus. 2019;11(2):e4024. doi:10.7759/cureus.4024.
10. Pastore F, Pastore A, Wittmann G, Hiddemann W, Spiekermann K, Winn RA. The role of therapeutic leukapheresis in hyperleukocytotic AML. PLoS One. 2014;9(4):7-10. doi:10.1371/journal.pone.0095062

11. Singh N, Lubana SS, Dabrowski L, Sidhu G. Leukostasis in chronic lymphocytic leukemia. Am J Case Rep. 2020;21:1-5. doi:10.12659/ AJCR.924798

12. Röllig C, Ehninger G. How I treat hyperleukocytosis in acute myeloid leukemia. Blood. 2015;125(21):3246-3252. doi:10.1182/blood2014-10-551507

13. Porcu P, Farag S, Marcucci G, Cataland SR, Kennedy MS, Bissell M. Leukocytoreduction for acute leukemia. Ther Apher. 2002;6 (1):15-23. doi:10.1046/j.1526-0968.2002.00402.x

14. Ventura GJ, Hester JP, Smith TL, Keating MJ. Acute myeloblastic leukemia with hyperleukocytosis: risk factors for early mortality in induction. Am J Hematol. 1988;27(1):34-37. doi:10.1002/ajh.2830270109

15. Giles FJ, Shen Y, Kantarjian HM, et al. Leukapheresis reduces early mortality in patients with acute myeloid leukemia with high white cell counts but does not improve long- term survival. Leuk Lymphoma. 2001;42(1-2):67-73. doi:10.3109/10428190109097677

16. Bug G, Anargyrou K, Tonn T, et al. Impact of leukapheresis on early death rate in adult acute myeloid leukemia presenting with hyperleukocytosis. Transfusion (Paris). 2007;47(10):1843-1850. doi:10.1111/j.1537-2995.2007.01406.x

17. Malkan UY, Ozcebe OI. Leukapheresis do not improve early death rates in acute myeloid leukemia patients with hyperleukocytosis. Transfus Apher Sci. 2017;56(6):880-882. doi:10.1016/j.transci.2017.11.002

18. Shallis RM, Stahl M, Wei W, et al. Patterns of care and clinical outcomes of patients with newly diagnosed acute myeloid leukemia presenting with hyperleukocytosis who do not receive intensive chemotherapy. Leuk Lymphoma. 2020;61(5):1220-1225. doi:10.1080/10428194.2020.1728753

19. Nan X, Qin Q, Gentille C, et al. Leukapheresis reduces 4-week mortality in acute myeloid leukemia patients with hyperleukocytosis-a retrospective study from a tertiary center. Leuk Lymphoma. 2017;58(9):2110-2117. doi:10.1080/10428194.2016.1277386

20. Malkan UY, Gunes G, Eliacik E, et al. The factors affecting early death after the initial therapy of acute myeloid leukemia. Int $J$ Clin Exp Med. 2015;8(12):22564-22569.

21. Stahl M, Shallis RM, Wei W, et al. Management of hyperleukocytosis and impact of leukapheresis among patients with acute myeloid leukemia (AML) on short- and long-term clinical outcomes: a Large, Retrospective, Multicenter, International Study. Leukemia. 2020;34(12):3149-3160. doi:10.1038/s41375-020-0783-3

22. Bewersdorf JP, Zeidan AM. Hyperleukocytosis and leukostasis in acute myeloid leukemia: can a better understanding of the underlying molecular pathophysiology lead to novel treatments? Cells. 2020;9 (10):2310. doi:10.3390/cells9102310

23. Stucki A, Rivier A-S, Gikic M, Monai N, Schapira M, Spertini O. Endothelial cell activation by myeloblasts: molecular mechanisms of leukostasis and leukemic cell dissemination. Blood. 2001;97 (7):2121-2129. doi:10.1182/blood.V97.7.2121

24. McKee LC, Collins RD. Intravascular leukocyte thrombi and aggregates as a cause of morbidity and mortality in leukemia. Medicine (Baltimore). 1974;53(6):463-478. doi:10.1097/00005792-197411000-00006

25. Zuckerman T, Ganzel C, Tallman MS, Rowe JM. How I treat hematologic emergencies in adults with acute leukemia. Blood. 2012;120 (10):1993-2002. doi:10.1182/blood-2012-04-424440

26. Langleben D, Orfanos SE, Giovinazzo M, et al. Pulmonary capillary surface area in supine exercising humans: demonstration of vascular recruitment. Am J Physiol Lung Cell Mol Physiol. 2019;317(3): L361-8. doi:10.1152/ajplung.00098.2019

27. Cavenagh JD, Gordon-Smith EC, Gibson FM, Gordon MY. Acute myeloid leukaemia blast cells bind to human endothelium in vitro utilizing E-selectin and vascular cell adhesion molecule-1 (VCAM-1). Br J Haematol. 1993;85(2):285-291. doi:10.1111/ j.1365-2141.1993.tb03168.x 
28. Hölig K, Moog R. Leukocyte depletion by therapeutic leukocytapheresis in patients with leukemia. Transfus Med Hemother. 2012;39 (4):241-245. doi:10.1159/000341805

29. Jin Y, Guo S, Cui Q, et al. A hospital based retrospective study of factors influencing therapeutic leukapheresis in patients presenting with hyperleukocytic leukaemia. Sci Rep. 2018;8:294. doi:10.1038/ s41598-017-17534-4

30. Hoffman R, Benz E, Silberstein L, et al. Hematology: Basic Principles and Practice. 7th ed. Elsevier; 2017:924-953.

31. Davenport A, Tolwani A. Citrate anticoagulation for continuous renal replacement therapy (CRRT) in patients with acute kidney injury admitted to the intensive care unit. NDT Plus. 2009;2(6):439-447. doi:10.1093/ndtplus/sfp136

32. Shelat SG. Practical considerations for planning a therapeutic apheresis procedure. Am J Med. 2010;123(9):777-784. doi:10.1016/j. amjmed.2010.01.022

33. Schwartz J, Winters JL, Padmanabhan A, et al. Guidelines on the use of therapeutic apheresis in clinical practice-evidence-based approach from the writing committee of the American Society for apheresis: the sixth special issue. J Clin Apher. 2013;28(3):145-284. doi: $10.1002 /$ jca. 21276

34. Padmanabhan A, Connelly-Smith L, Aqui N, et al. Guidelines on the use of therapeutic apheresis in clinical practice - evidence-based approach from the writing committee of the American Society for apheresis: the eighth special issue. J Clin Apher. 2019;34 (3):171-354.

35. Makroo RN, Kakkar B, Chowdhry M, Agrawal S, Seth S, Thakur UK. Therapeutic leukapheresis in a tertiary care hospital: a case series. Asian J Transfus Sci. 2017;11(1):65-68. doi:10.4103/ 0973-6247.200772

36. Choi MH, Choe YH, Park Y, et al. The effect of therapeutic leukapheresis on early complications and outcomes in patients with acute leukemia and hyperleukocytosis: a Propensity Score-Matched Study. Transfusion (Paris). 2018;58(1):208-216. doi:10.1111/trf.14329

37. Göçer M, Kurtoğlu E. Effect of prophylactic leukapheresis on early mortality and overall survival in acute leukemia patients with hyperleukocytosis. Ther Apher Dial. 2021. doi:10.1111/17449987.13645

38. Bewersdorf JP, Giri S, Tallman MS, Zeidan AM, Stahl M. Leukapheresis for the management of hyperleukocytosis in acute myeloid leukemia-a systematic review and meta-analysis. Transfusion (Paris). 2020;60(10):2360-2369. doi:10.1111/trf.15994
39. Triyono T, Hans V. Therapeutic apheresis in Asia: an Indonesia single center experience. J Clin Apher. 2014;28(April 2013):349-355.

40. de Jonge HJM, Valk PJM, de Bont ESJM, et al. Prognostic impact of white blood cell count in intermediate risk acute myeloid leukemia: relevance of mutated NPM1 and FLT3-ITD. Haematologica. 2011;96 (9):1310-1317. doi:10.3324/haematol.2011.040592

41. Padilha SL, Matos MCC, Domino NR, Domino NR. Acute myeloid leukemia: survival analysis of patients at a university hospital of Paraná. Rev Bras Hematol Hemoter. 2015;37(1):21-27. doi:10.1016/j.bjhh.2014.11.008

42. Liu C, Hong Y, Kuan AS, et al. The risk of early mortality in elderly patients with newly diagnosed acute myeloid leukemia. Cancer Med. 2020;9(4):1572-1580. doi:10.1002/cam4.2740

43. Kuo KHM, Callum JL, Panzarella T, et al. A retrospective observational study of leucoreductive strategies to manage patients with acute myeloid leukaemia presenting with hyperleucocytosis. $\mathrm{Br}$ J Haematol. 2015;168(3):384-394. doi:10.1111/bjh.13146

44. Kantarjian H, Ravandi F, O'Brien S, et al. Intensive chemotherapy does not benefit most older patients (age 70 years or older) with acute myeloid leukemia. Blood. 2010;116(22):4422-4429. doi:10.1182/ blood-2010-03-276485

45. Malfuson J-V, Etienne A, Turlure P, et al. Risk factors and decision criteria for intensive chemotherapy in older patients with acute myeloid leukemia. Haematologica. 2008;93(12):1806-1813. doi:10.3324/ haematol.13309

46. Malkawi A, Anand A, Al-Ameri A, Abdelfatah M, Kanaan Z, Haller N. Outcome of patients with acute myeloid leukemia/high risk MDS according to the kidney function. Blood. 2013;122 (21):5007. doi:10.1182/blood.V122.21.5007.5007

47. Dhakal P, Lyden E, Lee A, et al. Effects of obesity on overall survival of adults with acute myeloid leukemia. Clin Lymphoma Myeloma Leuk. 2020;20(3):e131-6. doi:10.1016/j.clml.2019.11.001

48. Navarro WH. Impact of obesity in the setting of high-dose chemotherapy. Bone Marrow Transplant. 2003;31(11):961-966. doi:10.1038/sj.bmt.1704052

49. Kendrick J, Warkentin D, Ensom MHH. The impact of obesity on pharmacokinetics and dosing of leukemia chemotherapy. In: Mittelman SD, Berger NA, editors. Energy Balance and Hematologic Malignancies [Internet]. Boston, MA: Springer US; 2012:97-127. Energy Balance and Cancer. doi:10.1007/978-1-46142403-1_5

\section{Publish your work in this journal}

The Journal of Blood Medicine is an international, peer-reviewed, open access, online journal publishing laboratory, experimental and clinical aspects of all aspect pertaining to blood based medicine including but not limited to: Transfusion Medicine; Blood collection, Donor issues, Transmittable diseases, and Blood banking logistics; Immunohematology; Artificial and alternative blood based therapeutics; Hematology; Biotechnology/nanotechnology of blood related medicine; Legal aspects of blood medicine; Historical perspectives. The manuscript management system is completely online and includes a very quick and fair peer-review system. Visit http://www.dovepress.com/testimonials.php to read real quotes from published authors. 